\title{
Genetic Heterogeneity of Murine Coronaviruses
}

\author{
By \\ M. M. C. LaI ${ }^{1}$, J. O. Fleming 1 , St. A. Stohlman ${ }^{1}$, and K. Fujrwara ${ }^{2}$ \\ 1 Departments of Microbiology and Neurology, \\ University of Southern California, School of Medicine, \\ Los Angeles, California, U.S.A., \\ and \\ 2 Department of Veterinary Pathology, School of Veterinary Science, \\ University of Tokyo \\ Tokyo, Japan \\ With 3 Figures
}

Accepted August 3, 1983

\begin{abstract}
Summary
Several mouse hepatitis viruses (MHV) with different pathogenicity were studied by oligonucleotide fingerprinting. Two strains, MHV-K and MHV-D, which were isolated in Japan and, which cause anaplasia and necrosis of bone marrow and diarrhea, respectively, were found to be closely related to MHVA59, the prototype MHV. Two other MHV strains, isolated from nude mice, were found to have diverged extensively from the known MHV strains. The MHVs isolated from separate cloned neuroblastoma cell lines persistently infected with JHM strain were also found to have diverged more markedly than the corresponding virus maintained under the conditions of lytic infection. Genetic divergence during persistent infection may be one of the mechanisms by which the MHV diverges.
\end{abstract}

\section{Introduction}

Mouse hepatitis virus (MHV), a member of the Coronaviridae, is an RNA virus containing a gingle-stranded RNA genome of $5.4 \times 10^{6}$ molecular weight (12). It has been isolated from mice in many parts of the world and has been associated mainly with hepatitis and encephalitis $(2,21)$. It replicates in a variety of tissue culture cells of rodent origin, including fibroblasts and neuronal cells (15). MHV infection in vitro generally results in the lysis of infected cells, but can also lead to persistent infection $(3,7,19)$. In nature, the virus survives by establishing latent infections in the gastrointestinal tract of mice (21). Viruses of this group have also been shown to cause enteric infections and wasting diseases in nude 
mice (20). Thus, the various strains of mouse hepatitis virus exhibit a wide range of pathogenic potentials.

We have previously studied several MHV strains by oligonucleotide fingerprinting, and found that they were widely divergent (13). However, some strains are much more closely related, e.g. MHV-A59 and MHV-3, suggesting a close genetic origins (13). This approach is useful in determining the evolutionary relationship among various MHV strains. In this study, we have further examined several mouse hepatitis virus strains isolated in Japan. Two of these viruses revealed extensive genetic homology with the prototype MHV-A59 strain. Thus, these strains appear to have remained genetically stable in nature. We have also compared the JHM strain of MHV (MHV-JHM) passaged under the conditions of lytic infection with those maintained as persistent infections in vitro. The viruses maintained under the latter conditions exhibit much more variability, suggesting that persistent infections may be a mechanism contributing to the genetic heterogeneity of the current MHV strains.

\section{Materials and Methods}

\section{Viruses and Cells}

The MHV-D strain was isolated from an infant mouse with fatal diarrhea, and has been shown to cause hepatitis and diarrhea in suckling mice $(4,8,9)$. MHV-K was isolated from an asymptomatic adult nude mouse $(\mathrm{nu} / \mathrm{nu}, \mathrm{Balb} / \mathrm{C})$. This strain causes bone marrow anaplasia and necrosis as well as hepatosplenic hemopoiesis (10). MHV. $\mathrm{NuU}$ was isolated from a nude mouse with wasting syndrome and hepatitis (5), and causes fatal wasting disease in nude mice but is not pathogenic in euthymic mice (20). These three strains were all isolated in Japan. MHV-M was isolated from a nude mouse and was a generous gift of Dr. Michael Collins, Microbiological Associates, Bethesda, Maryland. The two MHV-A59 strains studied were originally obtained from Dr. C. Bond of the University of California at San Diego and from Dr. K. Holmes of the Uniformed Services University, Bethesda, Maryland. All viruses were grown in DBT cells as previously described (13).

The in vitro persistent infection was established by growing MHV-JHM in a mouse neuroblastoma cell line (19). Single cell clones were obtained in the presence or absence of anti-JHM hyperimmune ascitic fluid. Two of the viruses were rescued from the nonproducer cell clones by fusing with DBT cells in the presence of polyethylene glycol (18). These two viruses, designated CS 1 and CS 3, were found to be cold-sensitive mutants (17). Two other virus strains, A 28 and A 33, were obtained from the virusproducing persistently infected cell clones.

\section{Radiolabeling and Purification of Virus}

The same procedures as described by LaI and Stomumar (13) were followed. The ${ }^{32} \mathrm{P}$-labeled virus was extracted with phenol in the presence of 1 per cent SDS, and the RNA was prepared by separation on $10-25$ per cent sucrose gradients in an SW 55 rotor at 50,000 $\mathrm{rpm}$ for 1 hour. The $60 \mathrm{~S}$ RNA peak was collected and used for oligonucleotide fingerprinting.

\section{$T_{1}$-Oligonucleotide Fingerprinting}

The analysis of ${ }^{2} \mathrm{P}$-labeled $60 \mathrm{~S}$ RNA by two-dimensional polyacrylamide gel electrophoresis followed essentially the same procedures as described previously (11, 13). 


\section{Results}

\section{Oligonucleotide Fingerprinting of MHV Strains Causing Different Diseases}

To study the genetic relationship of different MHV strains causing various diseases in mice, we first examined the oligonucleotide fingerprints of two of the MHVs isolated in Japan. One, MHV-D, was isolated from a suckling mouse with fatal diarrhea (9) and could eause a similar disease in experimentally infected infant mice (8). The other, MHV-K, causes anaplastic and necrotic lesions in bone marrow of mice (10). The 32P-labeled $60 \mathrm{~S}$ RNAs of these two viruses were digested with RNase $\mathrm{T}_{1}$, and separated by two-dimensional polyacrylamide gel electrophoresis. As shown in Fig. 1, these two viruses have very similar oligonucleotide fingerprints, suggesting a close genetic origin. Most surprisingly, the fingerprints of MHV-D (Fig. 1B) is indistinguishable from that of MHV-A59 (Fig. 1C), the prototype $\mathrm{MHV}$, which we have studied previously (13). These data suggest that the MHV-D might have been derived from the MHV-A59 strain quite recently. The oligonucleotide fingerprint of MHV.K (Fig. 1A) was also similar to that of MHV-A59, but contained six additional oligonucleotides which were not present in MHV-D or MHV-A59. The very high extent of homology between MHV-K and MHV-A59 suggests that MHV-K is also a recent derivative of $\mathrm{MHV}-\mathrm{A} 59$. The finding that $\mathrm{MHV}-\mathrm{K}$ contained six more large $\mathrm{T}_{1}$-oligonucleotides than MHV-A59 suggests either that MHV-K has greater genetic complexity than MHV-A59 or that MHV-K is heterogeneous. To distinguish between these two possibilities, several subclones of MHV-K were plaquepurified and analyzed by oligonucleotide fingerprinting. Such an analysis showed that all of the MHV subclones are similar but not identical, suggesting that the virus is indeed heterogeneous; however, all of them are very similar to MHV-A59 (data not shown).

The MHV-A59 strain used here for comparison is the same strain we have examined previously (13). It has relatively weak pathogenicity (14). No clinically apparent symptoms were induced by the intracranial injection of 1000 plaqueforming units of MHV-A59 into mice (data not shown). However, the MHV-A59 strains maintained in other laboratories have much higher hepatotropic properties, inducing very severe hepatitis (K. HoLmEs, personal communication). We therefore compared the MHV-A59 strains obtained from different laboratories. We found that their oligonucleotide fingerprints are indistinguishable (data not shown). Therefore, these two MHV-A59 strains are essentially identical, or have only very minor differences that could not be detected by this technique.

\section{Oligonucleotide Fingerprints of $\mathrm{MHVs}$ Isolated from Nude Mice}

Several MHVs have been isolated from latently infected nude mice which had developed wasting syndrome. These virus strains are of relatively low virulence in euthymic mice but produced fatal wasting diseases in nude mice (20). In order to assess the genetic origin and the pathogenic potentials of these viruses peculiar to nude mice, we examined the oligonucleotide fingerprints of two of these isolates: one, designated NuU, was isolated in Japan (5) and the other, MHV-M, isolated in the United States (M. Collins, personal communication). As shown in Fig. 2, these two strains have entirely different oligonucleotide fingerprints. Furthermore, 


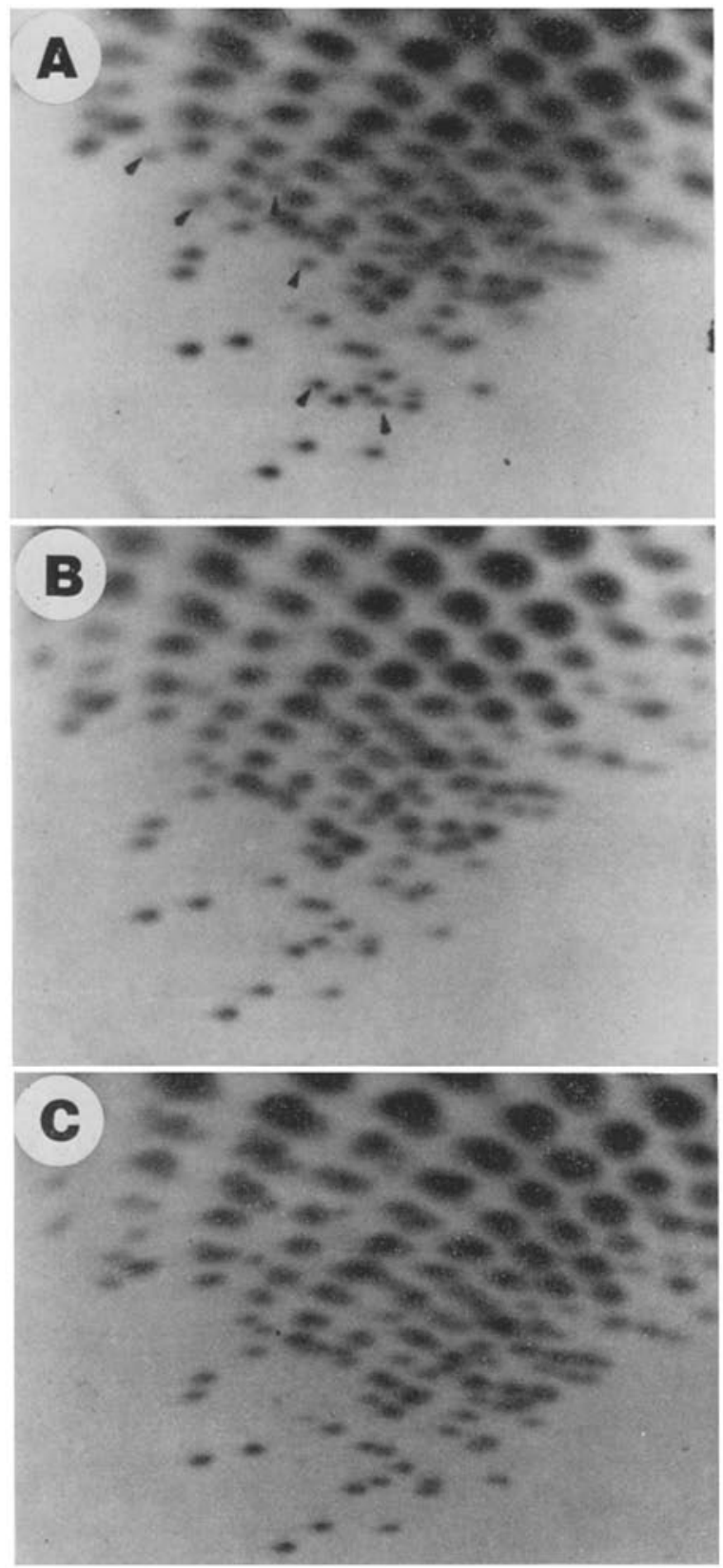

Fig. 1. Oligonueleotide fingerprints of various MHV strains. $A$ MHV-K $B$ MHV-D and $C$ MHV-A 59. The oligonucleotides denoted by triangles are those present only on MHV-K but not on MHV-A 59 
comparison with other known MHV isolates (13) revealed that they were also very distinct from any other MHV strains studied so far. Thus, these viruses represent quite unique MHV isolates.

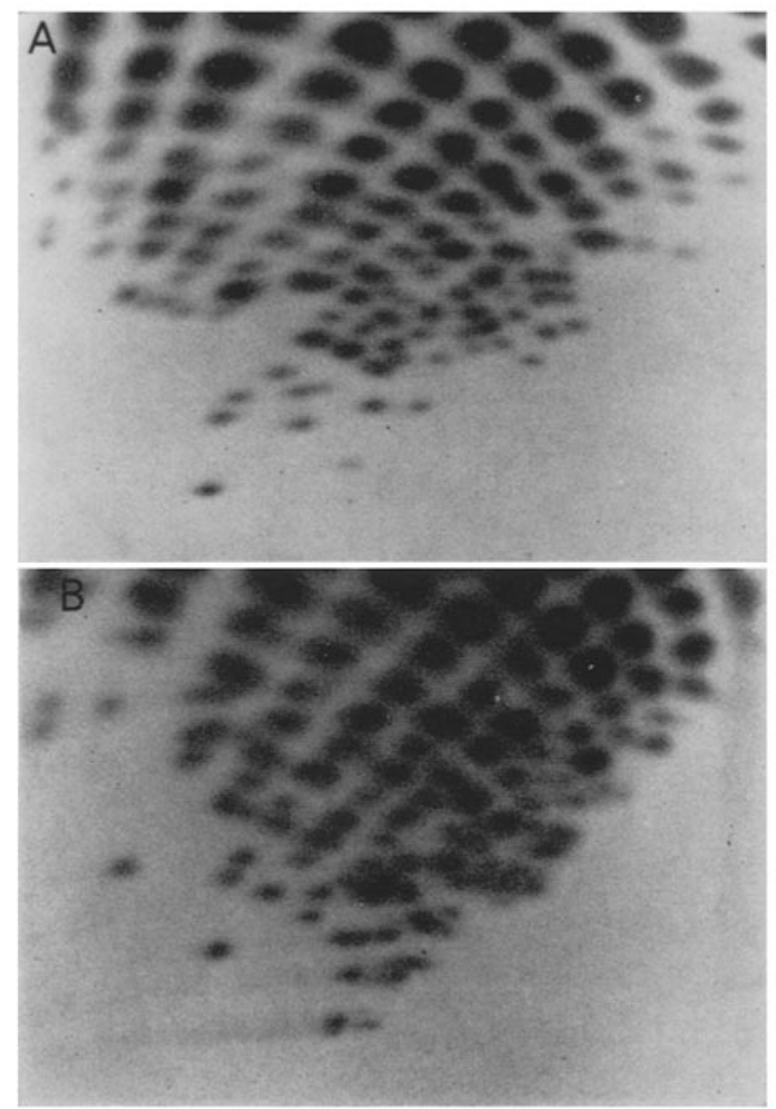

Fig. 2. Oligonucleotide fingerprints of MHVs isolated from nude mice. $A$ MHV-NuU and $B$ MHV-M

\section{Oligonucleotide Fingerprints of MHV.JHM Passaged in vitro}

To assess the genetic stability and variability of MHV and to study the possible mechanism of viral divergence observed as above, we examined the heterogeneity of viral genomic sequences in viruses isolated from persistently and latently infected cells. The persistent infection was established by infection of a mouse neuroblastoma cell line with the JHM strain of MHV (19). The viruses were rescued from separate cell lines derived from single cell clones by polyethylene glycol-mediated fusion with DBT or $17 \mathrm{CL} 1$ cells (18). Two of these rescued viruses, CS 1 and CS3, have been shown to be cold-sensitive (17). As shown in Fig. 3, the oligonucleotide fingerprints of both CS1 (Fig. 3B) and CS3 (Fig. 3C) are very similar to that of the small-plaque strain of the parental JHM (JHMDS) (Fig. 3A) (16). However, they both contain some oligonucleotides which are 


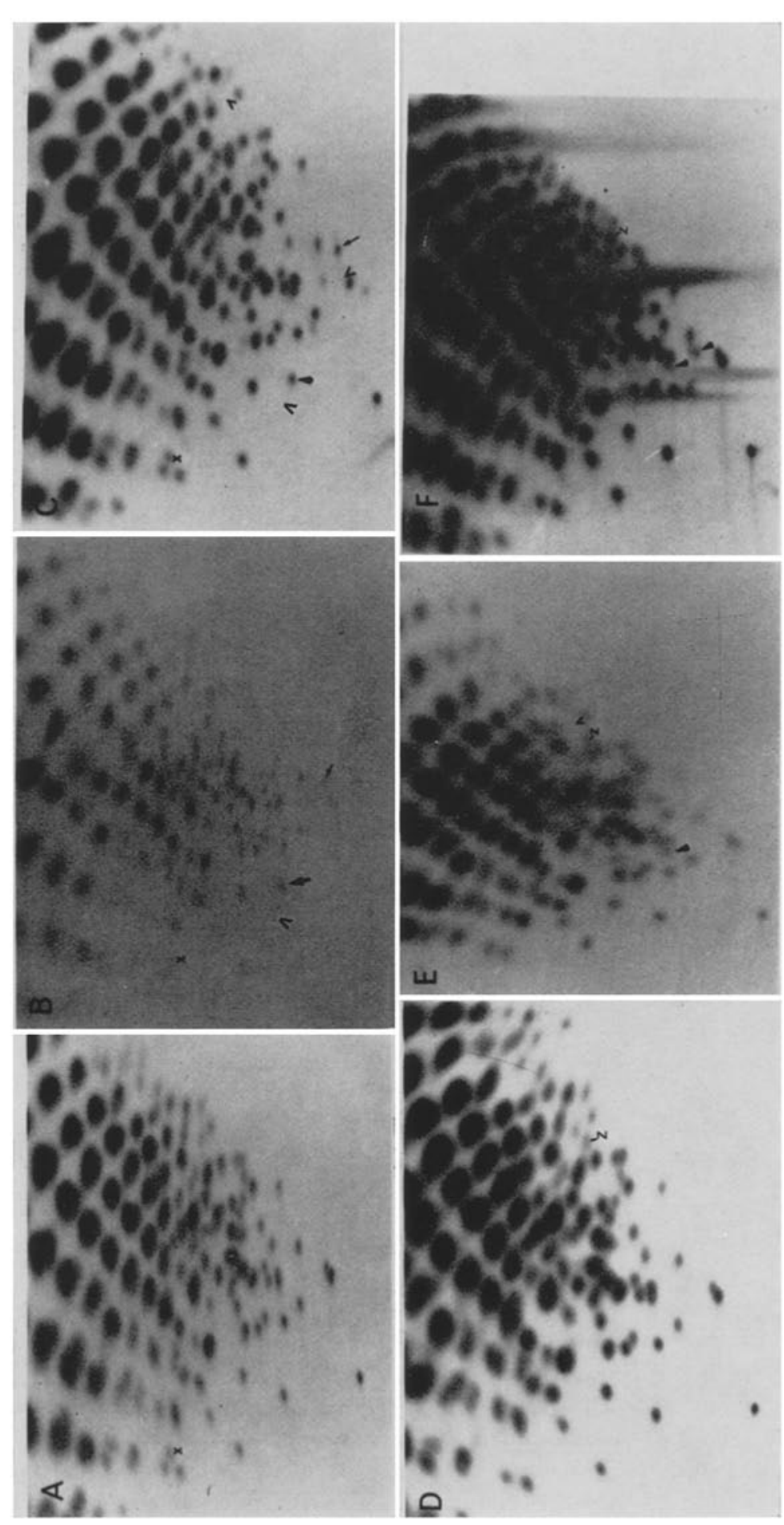

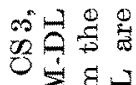

○멸

की

82.

内象兽

क⿺辶

实.䏝步

IN画

500

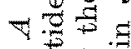

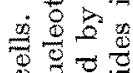

$\mathcal{3}$.

용. 응

5․ㅡㅇ 엉

然告

웅

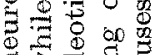

$\stackrel{2}{\circ} \cdot 9$

क्ष हैं

8 :

马

20 .

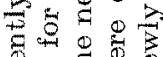

동

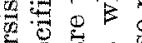

क

2 , w

g.

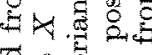

总电吉 8

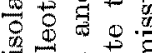

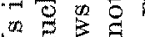

륭

폴

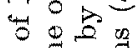

垫

5 is

2क⿺

क्षे

政的

도용

象

8 我

उ

要闲 80.9

$80,7=1$

흐욜

क

0.50
00 
unique for each strain. In addition, CS3 does not contain three oligonucleotides present in the JHM-DS. This result suggests that these two viruses represent the variants of JHM-DS, which were selected during persistent infection. Two other viruses, A28 (Fig. 3E) and A33 (Fig. 3F), were produced spontaneously from two different persistently infected neuroblastoma cell clones. These two viruses have oligonucleotide fingerprints similar to that of a large-plaque variant of JHM (JHM-DL), i.e. they contain a unique spot $z$, but lack the spot $x$, the latter of which is characteristic of JHM-DS (Fig. 3A; 16). However, they also contain at least one unique oligonucleotide spot not seen in the oligonucleotide fingerprints of either JHM-DL or JHM-DS, suggesting that these two viruses are derivatives of JHM-DL.

The isolation of JHM-DS and JHM-DL variants from the persistently infected cells was in sharp contrast with the viruses isolated under the conditions of lytic infection. As we have shown previously, MHV-JHM produced plaques of varying size on DBT cells (16). We have randomly picked at least 15 plaques on DBT cells and analyzed them by oligonucleotide fingerprinting. Only two fingerprint patterns were observed, one with the DS-type fingerprint and the other with the DL-type (16). The JHM-DS was subsequently serially passaged for at least 35 passages on DBT cells via lytic infection. The cloned viruses were studied at passages 6 and 35. The oligonucleotide fingerprints of the viruses passaged 35 times were exactly identical to that of JHM-DS (data not shown). No viruses of other genotypes were isolated, suggesting that MHV viruses are relatively stable during lytic infection, whereas persistent or latent infection in the meuroblastoma cell line tends to select for various types of variants.

\section{Diseussion}

We have previously studied six strains of mouse hepatitis virus isolated at different geographical locations and found them to be genetically heterogeneous (13). In this report, we have analyzed additional MHV strains, some of which were isolated in Japan. One surprising finding is the genetic similarity of those strains isolated in Japan to the MHV-A59 strain, the MHV prototype. These strains might, therefore, represent very recent derivatives of MHV-A59 or vice versa. This finding also suggests that these strains have remained relatively stable during passages through animals. It is interesting to note that these genetically highly related viruses have different pathogenicity: the MHV-A59 used in our laboratory is weakly pathogenic (14), MHV-D causes diarrhea (8), MHV-K causes bone marrow diseases (10), while another closely related strain MHV-3 causes severe hepatitis (13). Some of the genetic differences observed among these strains might be associated with the pathogenicity of the respective viruses. However, it is equally possible that these differences are not related to viral pathogenicity. In this regard, it is worth noting that several strains of MHV-A 59 have different pathogenicities and yet they could not be distinguished by oligonucleotide fingerprinting. It is possible that there are minor genetic differences among these viruses or that the different pathogenicity was contributed by host factors. This issue requires complete nucleotide sequence analysis of these and additional MHV strains. 
The finding that the viruses rescued from the persistently infected cells were more heterogeneous than those passaged under the conditions of lytic infection is similar to the observation with vesicular stomatitis virus (6). In that case, the VSV underwent progressive genetic changes as the cells were passaged in vitro (6). Similar long-term culturing of cells infected with other MHV strains has not been carried out; nevertheless, it is remarkable that the viruses rescued by cell fusion or produced spontaneously from these persistently infected cells are different from the parental viruses, while the randomly selected viruses maintained under the conditions of lytic infection are genetically stable. It is unclear why these cell lines would select for these unusual virus variants. It is noteworthy, however, that these variants produce lytic infection upon reinfection of the neuroblastoma cell line, and preliminary data indicated that these viruses do not produce diseases different from those induced by the parental viruses (S. A. STOHLMAN, unpublished results). Whether the selection of such variants in vivo contributes to the pathogenicity of the viruses is not clear at the present time.

The propensity of the persistently infected cells to select for viral mutants might be one of the mechanisms by which the MHV diverges. It is also noteworthy that the two viruses rescued by fusion of latently infected cell clones with the permissive cells were the derivatives of JHM-DS, while the viruses spontaneously produced by the persistently infected cells were JHM-DL derivatives. These two viruses, i.e. DS and DL, differ in their ability to cause chronic demyelination (16). There might be a correlation between the DL and the DS viruses and the ability to yield virus progeny under these conditions. Such a possibility is being studied in our laboratories.

\section{Acknowledgment}

We thank the excellent technical assistance of Todd Lasman, Todd Kennell, and Chris Patton. Wo also wish to thank Claudia Troesch, Alisa Young, and Raymond Mitchell for excellent editorial assistance. This work was supported by a research grant 1449-A from the National Multiple Sclerosis Society.

\section{References}

1. Calisher, L. S., Rowe, W. P.: Mouse hepatitis, Reo-3 and Theiler viruses. Nat. Cancer Inst. Monogr. 20, 67-75 (1966).

2. Gledhild, A. W., Niven, J. S. H.: Latent virus as exemplified by mouse hepatitis virus (MHV). Vet. Rev. Annot. 1, $82-90$ (1955).

3. Hirano, N., Goto, N., Makino, S., Fujrwara, K.: Persistent infection with mouse hepatitis virus JHM strain in DBT cell culture. Adv. in Exp. Med. and Biol. 142, $301-308(1981)$.

4. Hirano, N., Miyajima, H. Fujiwara. K.: Isolation of low-virulent mouse hepatitis virus from feces in infected mouse breeding colony. Jpn. J. Vet. Sci. 41, $31-40(1979)$.

5. Hirano, N., Tamura, T., Taguchi, F., Ueda, F., Fujiwara, K.: Isolation of low-virulent mouse hepatitis virus from nude mice with wasting syndrome and hepatitis. Jpn. J. Exp. Med. 45, 429-432 (1979).

6. Holland, J. J., Grabau, E. A., Jones, C. L., Semler, B. L. : Evolution of multiple genome mutations during long-term persistent infection by vesicular stomatitis virus. Cell 16, 495-504 (1979). 
7. Holmes, K. V., BeHNKe, J. N.: Evolution of a coronavirus during persistent infection in vitro. Adv. in Exp. Med. and Biol. 142, 287-300 (1981).

8. Ismida, T., FuJIWARA, K.: Pathology of diarrhea due to mouse hepatitis virus in the infant mouse. Jpn. J. Exp. Med. 49, 33-41 (1979).

9. Ismida, T., Tagucm, F., Lee, Y. S., Yamura, T., Fujrwara, K.: Isolation of mouse hepatitis virus from infant mice with fatal diarrhea. Lab. Animal Sci. 28, $269-276(1978)$.

10. Ishida, T., Tamura, T., UEDA, K., FuJIWara, K.: Hepatosplenic myelosis in naturally occurring mouse hepatitis virus infection in the nude mouse. Jpn. J. Vet. Sci. 40, 739-743(1978).

11. Lax, M. M. C., Brayton, P. R., Armen, R. C., Patton, C. D., Pugh, C., Stohliman, S. A.: Mouse hepatitis virus A $59: \mathrm{mRNA}$ structure and genetic localization of the sequence divergence from hepatotropic strain MHV-3. J. Virology 39, 823-834 (1981).

12. LaI, M. M. C., Stohlman, S. A.: The RNA of mouse hepatitis virus. J. Virology 26, $236-242(1978)$.

13. Lat, M. M. C., Stohlmar, S. A.: Comparative analysis of RNA genomes of mouse hepatitis viruses. J. Virology 38, 661-670 (1981).

14. RoBb, J. A., Bond, C. W.: Pathogenic murine coronaviruses. I. Charaeterization of the biological behavior in vitro and virus-specific intracellular RNA of strongly neurotropic JHMV and weakly neurotropic A $59 \mathrm{~V}$ viruses. Virology 94, 352-370 (1979).

15. RoBB, J.A., Bond, C. W.: Coronaviridae. In: Comprehensive Virology, Vol. 14 (Frakintel-Conrat, H., Waqder, R. R., eds.), 193-247. New York: Plenum Press Co.

16. Stohlman, S. A., Brayton, P. R., Fleminc, J. O., Weiner, L. P., Lai, M. M. C.: Murine coronaviruses: Isolation and characterization of two plaque morphology variants of the JHM neurotropic strain. J. gen. Virology 63, 265-275 (1982).

17. Stohlman, S. A., Sakaguchi, A. Y., Weiner, L. P.: Characterization of the coldsensitive murine hepatitis virus mutants rescued from latently infected cells by fusion. Virology 98, 448-445 (1979).

18. Stohlman, S. A., Sakagudhi, A. Y., Weiner, L. P.: Rescue of a positive-stranded RNA virus form antigen-negative neuroblastoma cells. Life Sci. 24, 1029-1036 (1979).

19. Stohtman, S. A., Werner, L. P.: Stability of neurotropic mouse hepatitis virus (JHM strain) during chronic infection of neuroblastoma cells. Arch. Virol. 57, $53-61(1978)$.

20. Taugra, T., Taguohi, F., Uena, K., FuJrwara, K.: Persistent infection with mouse hepatitis virus of low virulence in nude mice. Microbiol. Immunol. 21, $683-691$ (1977).

21. Wege, H. Sidpeld, S., Ter Meulew, V.: The biology and pathogenesis of coronaviruses. Curr. Top. Microbiol. Immunol. 99, 165-200 (1982).

Authors' address: Dr. M. M. C. LAI, Departments of Microbiology and Neurology, University of Southern California School of Medicine, Los Angeles, CA 90033, U.S.A.

Received June 22, 1983 